Journal of

Molecular Microbiology

and Biotechnology
J Mol Microbiol Biotechnol 2010;19:189-197

DOI: $\underline{10.1159 / 000320699}$

\title{
Membrane Topology Analysis of the Escherichia coli Aromatic Amino Acid Efflux Protein YddG
}

\author{
Larisa G. Airich $^{a}$ Irina S. Tsyrenzhapova ${ }^{a}$ Olga V. Vorontsova ${ }^{b}$ \\ Alexey V. Feofanov ${ }^{b}$ Vera G. Doroshenko ${ }^{a}$ Sergey V. Mashko ${ }^{a, b}$ \\ ${ }^{a}$ Ajinomoto-Genetika Research Institute and ${ }^{b}$ Biological Department, Lomonosov Moscow State University, \\ Moscow, Russia
}

\section{Key Words}

$\beta$-Lactamase $\cdot$ Fluorescence microscopy $\cdot$ Inner membrane protein - Poles of the cell $\cdot$ Reporter protein - Translation

fusion $\cdot$ Transmembrane segment $\cdot \mathrm{ZsGreen}$ and the early results of other workers regarding the cytoplasmic location of the C-terminus of YddG. The pole-specific localization of YddG-ZsGreen in E. coli cells was detected by fluorescence microscopy. Copyright $\odot 2010$ S. Karger AG, Basel

\section{Introduction}

Bacterial inner membrane proteins (IMPs) represent an important class of polypeptides that are involved in a wide variety of cellular functions. Among the $\sim 4,500$ open reading frames in the Escherichia coli genome (http://www.ncbi.nlm.nih.gov), approximately 900 (20\%) are predicted to encode IMPs that belong to the helical bundle class of proteins [Díaz-Mejía et al., 2009]. These proteins span the membrane with different numbers of transmembrane (TM) helices. Because of their hydrophobic and amphiphilic nature, IMPs are difficult to study and to obtain high-resolution three-dimensional structures. Therefore, an accurate topology model describing the number of TM spans and their orientations relative to the lipid bilayer is an important characteristic of an IMP. Topology models are usually produced by sequence-based prediction, which is better to confirm by experimental approaches [Yuan et al., 2010]. A construc-

\section{KARGER}

Fax +41613061234 E-Mail karger@karger.ch www.karger.com

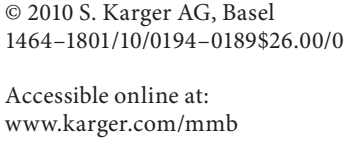

Prof. Sergey V. Mashko

Ajinomoto-Genetika Research Institute

1-st Dorozhny proezd, 1

RU-117545 Moscow (Russia)

Tel. +7 495780 3378, ext. 511, Fax +7 495315 0640, E-Mail sergey_mashko@ agri.ru 
tion of translation fusions of the studied IMP with a topological reporter molecule is usually used for this purpose. $\beta$-Lactamase (BlaM), green fluorescent protein (GFP) and alkaline phosphatase are able to act as topological reporter molecules when fused to different portions of an IMP [Broome-Smith et al., 1990; Manoil, 1991; Rapp et al., 2004].

YddG is an IMP that participates in the export of aromatic amino acids in E. coli [Doroshenko et al., 2007] and paraquat in Salmonella typhimurium [Santiviago et al., 2002]. Therefore, YddG is classified as belonging to the aromatic amino acid/paraquat exporter (ArAA/P-E) family (2.A.7.17) of the drug/metabolite transporter (DMT) (2.A.7) superfamily of proteins (M.H. Saier, www. tcdb.org). The size (293 aa) and primary structure of YddG, as the only protein of the ArAA/P-E family, most resembles the proteins of the $\mathrm{drug} / \mathrm{metabolite}$ exporter (DME) family (2.A.7.3) within the DMT. These proteins have 10 putative TM helices, but this has been experimentally shown only for PecM, a DME member [Rouanet and Nasser, 2001].

The location of the YddG C-terminus was earlier determined in topology mapping studies [Rapp et al., 2004]. Further experimental investigations of the structure of YddG are crucial for understanding its biological functions and properties and its possible applications in the metabolically engineered $E$. coli strains overproducing various aromatic compounds. In the present study, we investigated the general topology of the YddG membrane protein by constructing translational fusions of the Cterminal-truncated YddG with $\beta$-lactamase or Zoanthus sp. green fluorescent protein (ZsGreen) [Matz et al., 1999] reporters. The YddG-BlaM hybrids increased $\beta$-lactamase activity when the BlaM domain was located in the periplasmic space. In contrast, YddG-ZsGreen produced intensive fluorescence when the ZsGreen domain was located in the cytoplasmic compartment.

The obtained data unequivocally confirm the 10-TM helices-based membrane topology of YddG. Localization studies of YddG-ZsGreen showed enhanced fluorescence at the poles of $E$. coli cells.

\section{Results and Discussion}

\section{Construction and Analysis of the ydd $G^{\prime}-$ blaM Gene}

Fusions

In silico topological analysis of YddG has predicted the existence of 9- or 10-TM helices in this protein, depending on the software used: 9 according to TMPRED [Hof- mann and Stoffel, 1993], TM FINDER [Deber et al., 2001] and TopPred II [Claros and von Heijne, 1994], and 10 by SOSUI [Hirokawa et al., 1998] and TMHMM [Krogh et al., 2001] (fig. 1).

To clarify the membrane topological organization of YddG, $y d d G^{\prime}$-blaM fusions were created between exonuclease III-truncated $y d d G$ derivatives and the blaM gene encoding the leaderless $\beta$-lactamase. First, the recombinant plasmid pBRyddG-blaM was constructed based on pBR322 $\left(\mathrm{Ap}^{\mathrm{R}}, \mathrm{Tc}^{\mathrm{R}}\right)($ GenBank accession number J01749.1 [Sutcliffe, 1979]) as a vector. The $y d d G$ gene was inserted downstream of the $\mathrm{P}_{l a c}$ promoter and upstream of blaM. In the presence of excess IPTG (1 $\mathrm{mm}$ and up to $3 \mathrm{mM}$ ) in the culture medium, this recombinant plasmid conferred ampicillin resistance $\left(\mathrm{Ap}^{\mathrm{R}}\right)$ to $\sim 10 \mu \mathrm{g} / \mathrm{ml}$ and a high resistance to phenylalanine (up to $20 \mathrm{mg} / \mathrm{ml}$ ) to TG1 cells. Such a low level of $A^{R}{ }^{R}$ was consistent with a cytoplasmic location for the C-terminal portion of the hybrid YddG-BlaM protein [Rouanet and Nasser, 2001]. The resistance to phenylalanine was indicative of YddG functioning as an aromatic amino acid exporter [Doroshenko et al., 2007]. Therefore, the hybrid protein was integrated into the inner membrane with its C-terminal portion located in the cytoplasm. The decrease of the IPTG concentration to $0.1 \mathrm{~mm}$ resulted in decreases of $\mathrm{Ap}^{\mathrm{R}}$ and phenylalanine resistance. According to these results, it could also be proposed that overexpression of the YddG-BlaM fusion protein was not toxic to the bacterial cell even under conditions of a fully induced $\mathrm{P}_{l a c}$ promoter.

A set of internal deletions in pBRyddG-blaM was obtained by random partial digestion of the 3 '-terminus of the $y d d G$ gene (see 'Experimental Procedures'). The recombinant plasmids of interest were obtained by transforming TG1 cells and selecting tetracycline-resistant $\left(\mathrm{Tc}^{\mathrm{R}}\right)$ clones. Approximately $100 \mathrm{Ap}^{\mathrm{R}}$ clones from 500 tested $\mathrm{Tc}^{\mathrm{R}}$ clones were selected by growing on Luria-Bertani (LB) agar plates containing $1 \mathrm{mM}$ IPTG and $50 \mu \mathrm{g} /$ $\mathrm{ml}$ of ampicillin. This ampicillin concentration permitted the selection of only those clones with $\beta$-lactamase exported to the periplasm [Rouanet and Nasser, 2001]. The junctions of 12 randomly chosen $y d d G^{\prime}$-blaM fusions were sequenced. All of these fusions were located within the $y d d G$ coding region but were not evenly distributed. They were grouped into five regions corresponding to their periplasmic loops or their surrounding regions in the 10-TM helices topology model proposed by SOSUI and TMHMM (fig. 1b). No truncated variants of YddG conferred a high resistance to phenylalanine to E. coli cells. The level of ampicillin resistance for these $12 \mathrm{fu}$ - 


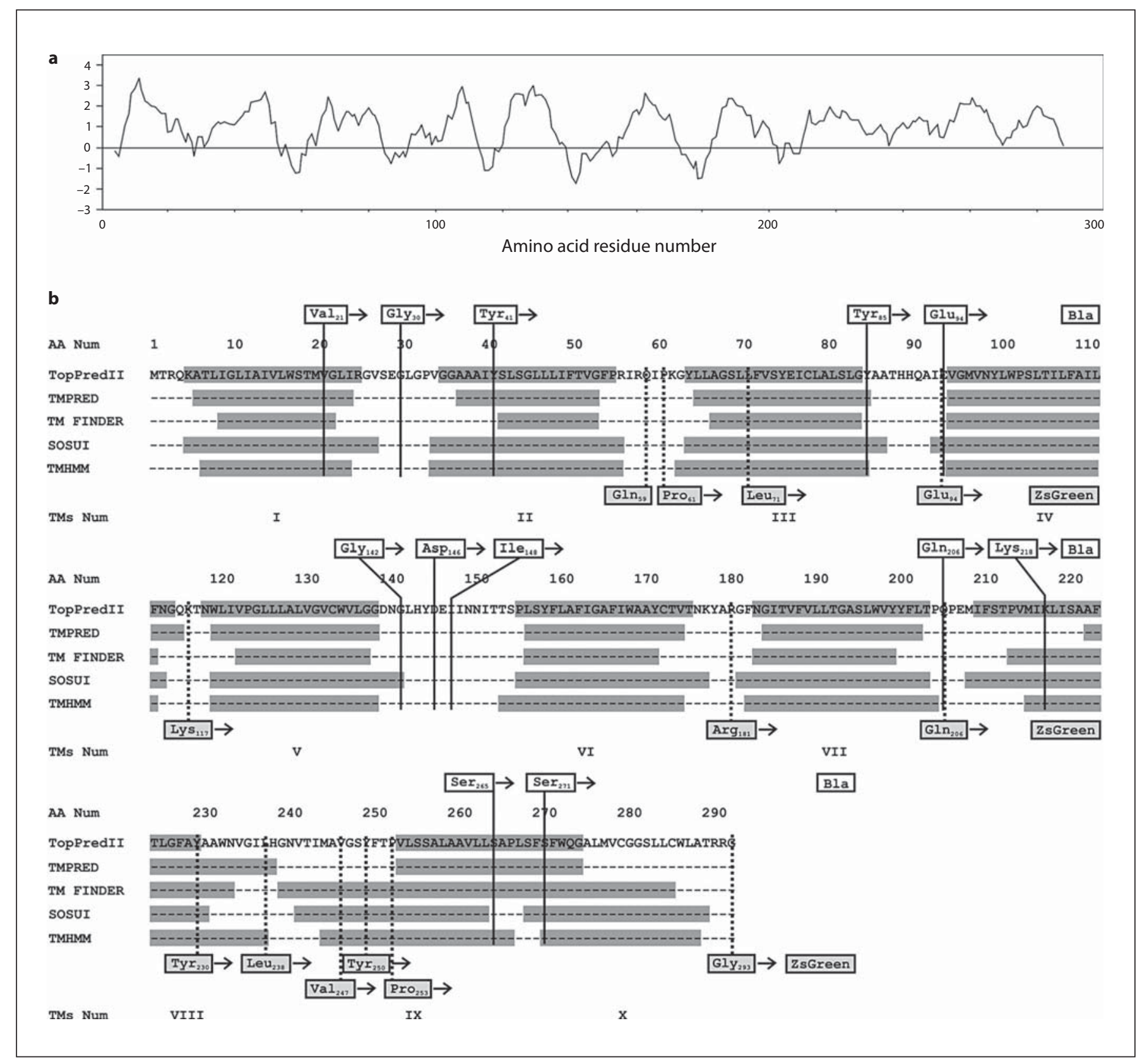

Fig. 1. a Hydropathy plot of YddG. The algorithm of Kyte and Doolittle [1982] was used with a window of nine residues. $\mathbf{b}$ YddG topology prediction from several computer programs. The gray rectangles correspond to putative TM helices. The positions of the designed YddG-BlaM and YddG-ZsGreen fusions are shown by solid and dashed lines, respectively.

sions was determined more precisely, and it was in the range of $75-200 \mu \mathrm{g} / \mathrm{ml}$ (fig. 2). The low ampicillin resistance, $75 \mu \mathrm{g} / \mathrm{ml}$, corresponded to the junctions of $\mathrm{Val}_{21}$, $\mathrm{Tyr}_{85}$, and $\mathrm{Lys}_{218}$ located in TMs I, III, and VIII. The higher ampicillin resistances, 200 and $150 \mu \mathrm{g} / \mathrm{ml}$, corre- sponded to the junctions of $\mathrm{Gly}_{30}$ and $\mathrm{Ser}_{265}$ located in the predicted periplasmic loops between TM helices I and II as well as between TM helices IX and X. The detection of these periplasmic loops confirmed the location of the $\mathrm{N}$ and C-termini of YddG in the cytoplasm. 


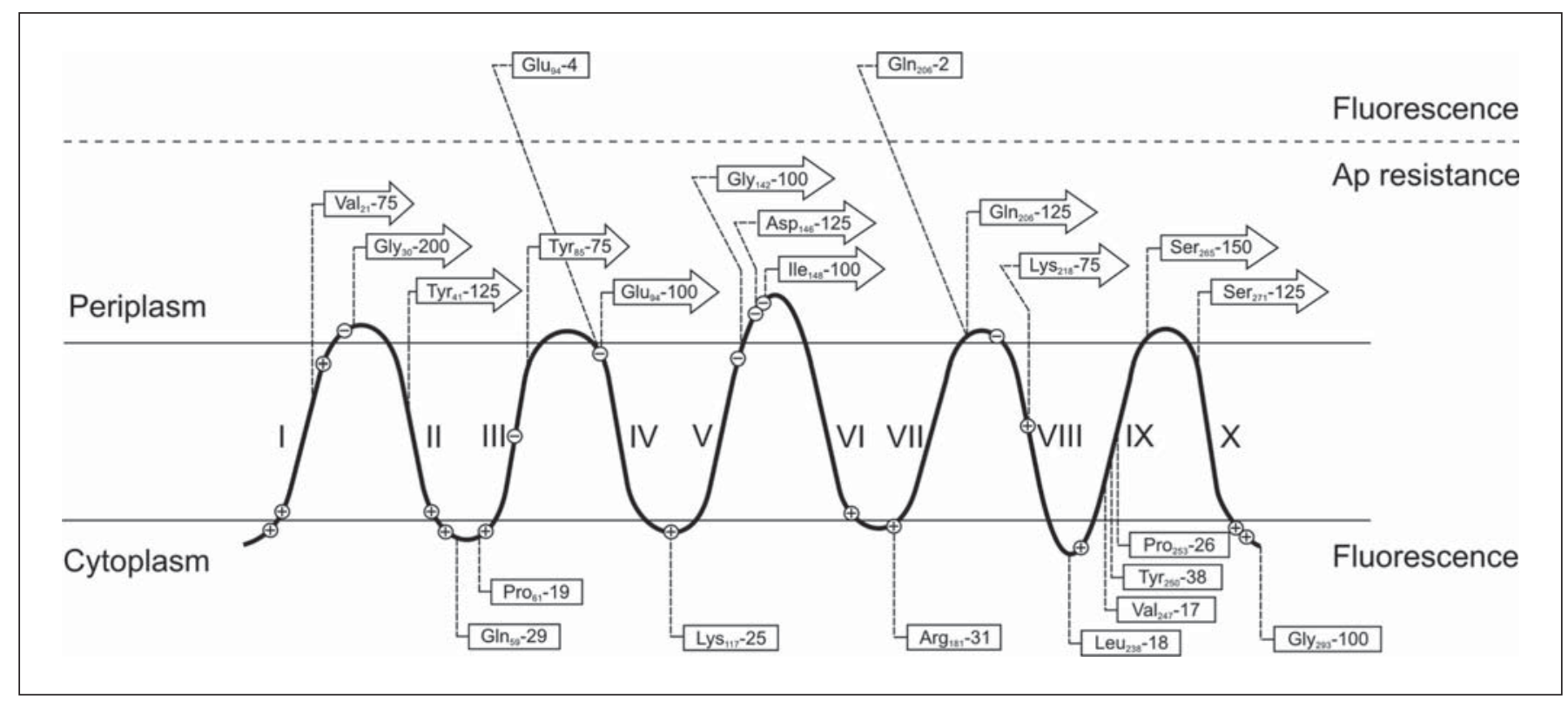

Fig. 2. Model for the topological organization of YddG based on the properties of the YddG-BlaM fusions, the hydropathy profile and the positive-inside rule [Von Heijne, 1986]. The positions at which BlaM and ZsGreen were fused to YddG are indicated by arrows and rectangles, respectively. The numerals inside the figures correspond to the number of the YddG amino acid residues and the level of ampicillin (Ap) resistance $(\mu \mathrm{g} / \mathrm{ml})$ for YddG-BlaM and to the level of fluorescence (\%) for YddG-ZsGreen. The positions of the charged Asp and Glu residues are shown by '-'; Arg and Lys residues are indicated by ' + '.

\section{Immunodetection of the YddG-BlaM Fusions}

To ensure that different levels of fusion protein expression did not affect the topological analysis, the accumulation of YddG-BlaM fusions in membrane extracts was semiquantitatively evaluated by Western blotting experiments using commercially available (Abcam) mouse antibodies to $\beta$-lactamase, as described in 'Experimental Procedures'. As can be seen in figure $3 \mathrm{a}$, all tested fusion proteins were expressed at similar levels. As compared with the standard set of reference soluble proteins, all fusions had greater mobilities in SDS-PAGE than those predicted from the calculated molecular masses, as usually reported for TM helices-based proteins [Rouanet and Nasser, 2001]. Nevertheless, the sizes of the hybrid proteins were compatible with the distribution of the fusion junctions along the $y d d G$ coding region. So, the detected difference in $\mathrm{Ap}^{\mathrm{R}}$ level for the strains with YddG-BlaM fusions manifested the features of the tested protein topologies.

\section{Construction and Analysis of the YddG-ZsGreen}

Fusions

To confirm the orientation of YddG in the inner membrane determined using the YddG-BlaM fusions, we made YddG-ZsGreen fusions, including a fusion consisting of a full-size YddG domain. All YddG-ZsGreen fusions were constructed by overlap extension PCR (see 'Experimental Procedures'). The plasmid pZsGreen (Clontech) was used as a template for amplification of the reporter gene. The fusion junctions were chosen in such a way as to test the presence of all four cytoplasmic loops predicted above. Three fusion points $\left(\mathrm{Gln}_{59}, \mathrm{Pro}_{61}\right.$ and $\mathrm{Leu}_{71}$ ) were chosen between TM helices II and III, fusion point Lys ${ }_{117}$ was between TM helices IV and V, and fusion point $\operatorname{Arg}_{181}$ was between TM helices VI and VII. Three fusion points ( $\mathrm{Val}_{247}, \mathrm{Tyr}_{250}$ and $\mathrm{Pro}_{253}$ ) were in TM helix IX because of the complicated hydrophobic plot in this region; thus, the cytoplasmic location of several amino acid residues could not be excluded (fig. 1a). The fusions $\mathrm{Glu}_{94}$ and $\mathrm{Gln}_{206}$ had the same points of YddG' junction with the reporter, serving as two 'periplasmic-positive' YddG-BlaM fusions and 'cytoplasmic-negative' YddGZsGreen controls. The strong fluorescence signal accepted as $100 \%$ was generated by the $\mathrm{Gly}_{293}$ fusion containing a full-size YddG (fig. 2). The plasmid with this fusion also conferred resistance to phenylalanine to $E$. coli cells, in contrast to truncated YddG-ZsGreen fusion variants. The cytoplasmic-negative controls, the fusions $\mathrm{Glu}_{94}$ and 


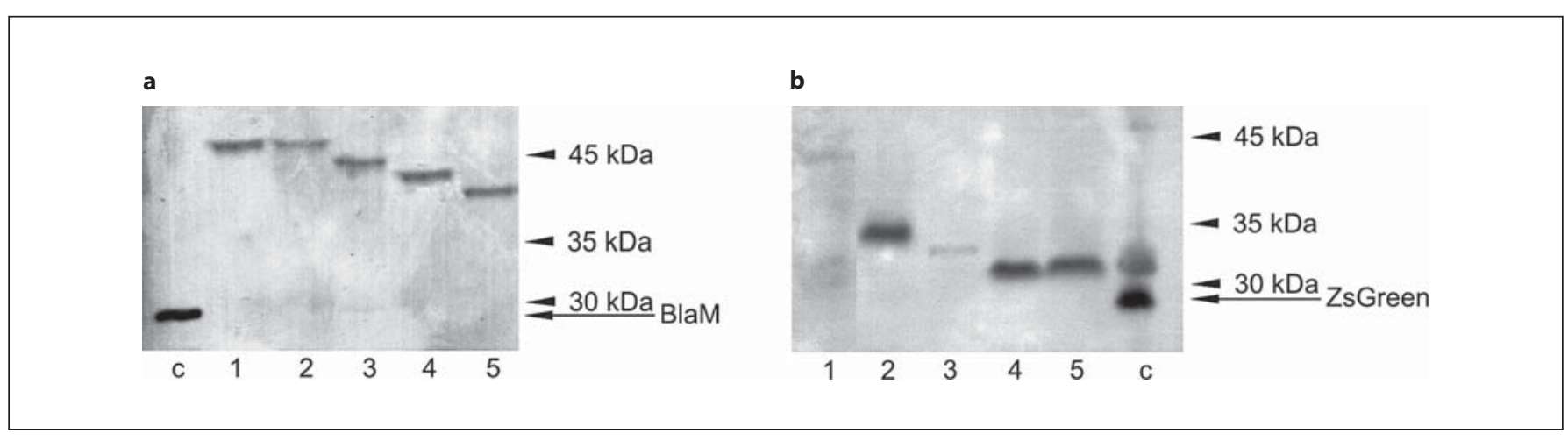

Fig. 3. Western blots of YddG-BlaM (a) and YddG-ZsGreen (b) fusions. Equal amounts of solubilized membrane fractions were separated by SDS-PAGE, and fusion proteins were visualized by immunoblotting with monoclonal antibodies against corresponding reporter proteins. The amino acids at which fusions oc- curred were as follows: $\mathbf{a} 1=\operatorname{Ser}_{271} ; 2=\operatorname{Ser}_{265} ; 3=\operatorname{Lys}_{218} ; 4=\operatorname{Gln}_{206}$; $5=\mathrm{Ile}_{148} ; \mathrm{c}($ control $)=$ cell extract of TG1 pBR322, expressing $\beta$ lactamase. $\mathbf{b} 1=\mathrm{Gln}_{206} ; 2=\operatorname{Arg}_{181} ; 2=\mathrm{Lys}_{11} ; 3=\mathrm{Glu}_{94} ; 4=\mathrm{PrO}_{61}$; $5=\operatorname{Gln}_{59} ; \mathrm{c}($ control $)=$ ZsGreen.
$\mathrm{Gln}_{206}$ with predicted periplasmic locations of the ZsGreen domain, showed the lowest signal, $\sim 3 \%$. Two fusions, $\mathrm{Leu}_{71}$ and $\mathrm{Tyr}_{230}$, generating strong signals of $\sim 100 \%$, appeared to be false positives because they were cleaved from the YddG protein and the released ZsGreen accumulated in the cytoplasm, as determined by Western blotting (data not shown) and by fluorescence microscopy (see below). The other YddG-ZsGreen fusions generated signals with fluorescence intensities of $\sim 20-30 \%$.

According to immunodetection of YddG-ZsGreen by Western blotting of the membrane protein fraction, all fusions were of the predicted sizes based on the locations of the junctions along the $y d d G$ gene. Fusion proteins with a cytoplasmic location of the ZsGreen domain were expressed at nearly the same levels (fig. 3b). Fusions $\mathrm{Glu}_{94}$ and $\mathrm{Gln}_{206}$, with ZsGreen domain in the periplasmic space, were weakly detectable in the membrane fraction. ZsGreen protein, as has been shown for GFP, was probably improperly folded when localized outside of the cytoplasm and was cleaved by proteases [Feilmeier et al., 2000]. Therefore, the obtained data concerning YddGZsGreen fusion proteins' fluorescence correlated well with the general 10-TM helices YddG topology predicted above using BlaM-tagged fusions.

The fluorescent signals of fusions $\mathrm{Val}_{247}, \mathrm{Tyr}_{250}$ and $\mathrm{PrO}_{253}$ within TM helix IX were typical of a cytoplasmic location of YddG' termini tagged by ZsGreen (these signals varied from 17 to $37 \%$ ). (Significant variations in the fluorescent signals of $\sim 10 \%$ were observed for two closer fusions, $\mathrm{Gln}_{59}$ and $\mathrm{Pro}_{61}$, localized in the first cytoplasmic loop as well; see fig. 1b.) Keeping in mind that in these fusions, ZsGreen is linked to rather short portions of TM helix IX, the fluorescence data could not exclude the possible complex topology of the native YddG in this region. To detail the YddG topology, more sophisticated methods are probably necessary.

\section{Intracellular Localization of the YddG-ZsGreen}

Hybrids as Assessed by Fluorescence Microscopy

To visualize the intracellular localization of YddGZsGreen, we inspected TG1 cells containing full-length YddG-ZsGreen fusion, fusions $\operatorname{Lys}_{117}, \operatorname{Arg}_{181}, \mathrm{Gln}_{206}$, and $\mathrm{Leu}_{238}$, as well as false fusion $\mathrm{Leu}_{71}$ using fluorescence microscopy. For the controls, cells of strains TG1 (pZsGreen) and TG1 (pBR322) were used. All of these plasmid-carrier strains were pregrown in LB medium in the presence of IPTG. The fluorescence signals were located at the poles of the cells containing full-length YddGZsGreen $\left(\mathrm{Gly}_{293}\right)$ and the fusions $\mathrm{Lys}_{117}, \mathrm{Arg}_{181}$, and $\mathrm{Leu}_{238}$. As can be seen from figure $4 \mathrm{a}$, d, where the results for $\mathrm{Gly}_{293}$ and $\mathrm{Arg}_{181}$ fusions are shown, the fluorescence was usually stronger at one (perhaps, 'old' [Brandon et al., 2003]) pole of the cells. Cells with practically equal intensities of fluorescence on both poles were also seen (fig. $4 \mathrm{~d}$ ). This is probably caused by a lag of YddG appearance at new poles after cell division.

Cells of the control strains, TG1 (pZsGreen) and TG1 (pBR322) (which carried the plasmid for native ZsGreen and the initial expression vector without any reporter gene), demonstrated either uniformly distributed fluorescence over the entire intracellular space (fig. 4b) or no fluorescence (fig. 4c), respectively. Cells containing the 

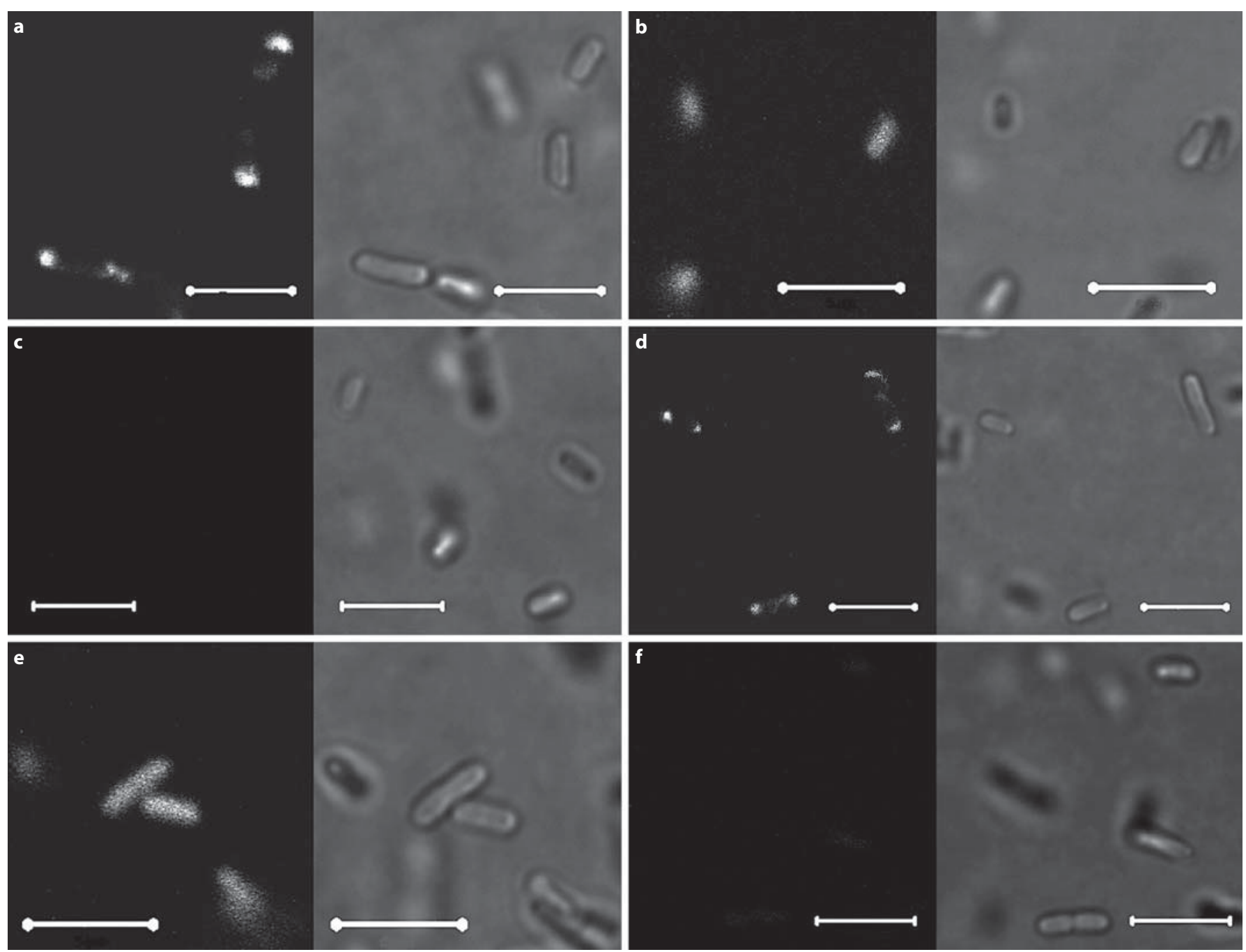

Fig. 4. Distribution of YddG-ZsGreen fusions in live E. coli TG1 cells. Confocal fluorescent (left panels) and bright-field (right panels) micrographs are shown. The plasmids in TG1 cells were as follows: a pBR-YddG(Gly $\left.{ }_{293}\right)$-ZsGreen. b pZsGreen. c pBR322. d pBR-YddG(Arg 181$)$-ZsGreen. e pBR-YddG(Leu $\left.{ }_{71}\right)$ ZsGreen. f pBR-YddG(Gln 206$)$-ZsGreen. The images were ob-

tained using an LSM510 META microscope that was equipped with a water immersion $63 \times / 1.2$ NA C-Apochromat objective. ZsGreen was excited at $488 \mathrm{~nm}$ and the emission light was detected using an HFT UV/488/543/633 MBS and an LP505 filter. Bars $=5 \mu \mathrm{m}$.

false-positive fusion $\mathrm{Leu}_{71}$ also demonstrated uniform fluorescence throughout the cell. Fluorescence was absent in the case of fusion $\mathrm{Gln}_{206}$, which had a periplasmic localization of the ZsGreen domain (fig. 4f).

Therefore, the fluorescence microscopy data confirm the membrane localization of YddG-ZsGreen fusions detected via Western blotting and indicate a polar localization of YddG. In the case of the false fusion $\mathrm{Leu}_{71}$, where the ZsGreen domain was cut off, fluorescence was distributed throughout the whole cell. The polar localization of YddG may be connected with a probable YidC-mediated

pathway of YddG biogenesis and insertion into the internal membrane [Kiefer and Kuhn, 2007; Xie and Dalbey, 2008]. In spite of the fact that the mechanism by which multispanning IMPs are inserted into the target membrane is not completely understood, two main machines of the Sec/YidC-translocon and the YidC insertase have been identified [reviewed in Kuhn, 2009; Luirink et al., 2005]. In fact, YidC insertase localizes predominantly to the poles of E. coli [Urbanus et al., 2002], whereas Secbased complexes are distributed throughout the circumference of the inner membrane [Brandon et al., 2003]. 


\section{Conclusions}

In this study, we investigated the topological organization of YddG in the E. coli inner membrane. YddG is the first E. coli amino acid exporter for which the topology was experimentally determined. The identification of this protein as a paralogue of RhtA and YdeD, which were formerly characterized as threonine and cysteine exporters, was followed by the finding of YddG's ability to export aromatic amino acids under conditions of their overproduction in cells [Doroshenko et al., 2007]. RhtA and YdeD are related to the DME family (2.A.7.3). The topology of YddG experimentally coincides with the previously determined 10-TM helices topology with cytoplasmic locations of $\mathrm{N}$ - and C-termini of the DME-family protein PecM. Moreover, global topological analysis of E. coli IMPs has revealed cytoplasmic locations of both termini as being the predominant topology of E. coli proteins [Daley et al., 2005].

Based on the information obtained from our study using YddG-ZsGreen fusion proteins, it may be possible to design new strategies to enhance YddG accumulation in the inner cell membrane. These strategies will most likely require the optimization of exporter gene expression, the targeting of the nascent IMP and its insertion into the E. coli inner membrane. These approaches will be available for many other exporters with a YddG-like type of biogenesis and will greatly improve the performance of metabolically engineered strains producing intracellular metabolites of commercial interest.

\section{Experimental Procedures}

Strains, Plasmids and Growth Conditions

The TG1 strain [K12 $\Delta$ (lac-pro), supE, thi, hsd $5 \mathrm{~F}^{\prime}(\mathrm{tra} \Delta 36$, pro $A B^{+}, \operatorname{lacI}^{q}$, lacZ $\left.\left.\Delta M 15\right)\right]$ was used as a recipient for plasmid constructions. All recombinant plasmids were constructed based on pBR322 [Bolivar et al., 1977; Sutcliffe, 1979]. The media used were LB, SOB or M9 minimal medium, with $0.4 \%$ glucose as the carbon source [Sambrook and Russell, 2001]. Solid media were prepared by adding agar $(15 \mathrm{~g} / \mathrm{l})$ to the liquid media. Antibiotics were used at the following concentrations: ampicillin at $100 \mu \mathrm{g} / \mathrm{ml}$ and tetracycline at $12.5 \mu \mathrm{g} / \mathrm{ml}$.

The resistance to phenylalanine (minimal inhibitory concentration) was determined as described previously [Doroshenko et al., 2007].

Plasmid pZsGreen encoding a variant of wild-type ZsGreen was purchased from Clontech. ZsGreen is an analog of the GFP from Aequorea victoria and a member of the reef coral fluorescent protein (RCFP) family. The ZsGreen monomer shares structural homology with GFP but, unlike GFP, can self-associate in solution to form dimers and tetramers. In spite of this feature, published studies have shown that ZsGreen may be used as a fusion tag [Heddle and Mazaleyrat, 2007].

\section{Construction of Plasmids}

Construction and restriction analysis of the recombinant DNA, agarose gel electrophoresis, $\mathrm{Ca}^{2+}$-dependent transformation and electroporation of $E$. coli cells were all carried out using standard experimental protocols [Sambrook and Russell, 2001]. Restriction endonucleases, T4 DNA ligase, exonuclease III, S1 nuclease and the Klenow fragment of E. coli DNA polymerase (Fermentas) were used according to the manufacturer's instructions. The sequences of all primers are available upon request.

In pBR322, blaM was inactivated by deleting the DraI restriction fragment (711 bp). Re-ligation yielded the plasmid pBR322 2 blaM. To construct the $y d d G$-blaM fusion, a DNA fragment containing the $y d d G$ gene was amplified from TG1 chromosomal DNA, and the $\mathrm{P}_{l a c}$ promoter was amplified by PCR from the ZsGreen plasmid. These two fragments were joined using overlapping PCR to create the $y d d G$ gene cassette downstream of the $\mathrm{P}_{\text {lac }}$ promoter. The DNA fragment containing a truncated version of blaM was amplified from the pBR322 plasmid and then linked with the $y d d G$ gene under the control of the $\mathrm{P}_{\text {lac }}$ promoter by overlapping PCR. The primers introduced Aat II and DraI sites to the 5 ' - and 3 '-ends, respectively, of the final DNA amplification product. NcoI and SacI restriction sites were also introduced in the $y d d G-b l a M$ fusion region for the construction of the truncated variants by exonuclease III treatment (see the following section). After restriction digestion by AatII and DraI, the obtained DNA fragment was purified and ligated into the AatII-DraI sites of the pBR322 2 blaM plasmid to yield pBRyddG-blaM.

Thirteen specific PCR products containing truncated $y d d G$ genes after the $\mathrm{P}_{l a c}$ promoter fused in frame with ZsGreen were obtained by overlapping PCR of two sets of fragments (the $y d d G^{\prime}$ fragments amplified from pBR $y d d G-b l a M$, and the ZsGreen fragment from pZsGreen). After restriction digestion by AatII and DraI, the fragments were cloned into the pBR322 $\Delta$ blaM plasmid, which yielded a set of $\mathrm{pBR} y d d G-Z s G r e e n$ plasmids. The structures of the obtained plasmids were confirmed by sequencing the YddG-ZsGreen fusion regions.

\section{Deletions with Exonuclease III and the yddG-blaM Gene}

Fusions

The plasmid pBRyddG-blaM was used to construct a series of gene fusions by nested deletions from the $3^{\prime}$-end of $y d d G$. Exonuclease III digests linear DNA possessing $5^{\prime}$ overhangs or blunt ends, but not $3^{\prime}$ overhangs. The NcoI-produced DNA end in pBRyddG-blaM was sensitive to this nuclease, whereas the SacIdriven end was resistant. After linearization of the plasmid by $\mathrm{NcoI}$ and SacI treatment, $12 \mathrm{mg}$ of the plasmid DNA was resuspended in $50 \mu \mathrm{l}$ of exonuclease III buffer and incubated at $30^{\circ} \mathrm{C}$. Exonuclease III (500 U in $2.5 \mu \mathrm{l}$ ) was then added. At this temperature, the exonuclease III digestion reaction proceeded at 200 bases/min. Aliquots $(2 \mu \mathrm{l})$ were removed at 45 -second intervals, immediately mixed with $7.5 \mu$ l of S1 nuclease ( $2 \mathrm{U}$ of S1 nuclease) and incubated on ice. After all the aliquots were collected, the tubes were removed from the ice and incubated at room temperature for $30 \mathrm{~min}$. The tubes were then incubated at $70^{\circ} \mathrm{C}$ for $10 \mathrm{~min}$ to deactivate the $\mathrm{S} 1$ nuclease. The samples were analyzed on a $1 \%$ agarose gel. The digestion products were purified, end-filled with deoxyribonucleotides $(10 \mathrm{mM})$ by the Klenow fragment of E. coli 
DNA polymerase I ( $1 \mathrm{U} / \mu \mathrm{g}$ of DNA) and then ligated to the pBR322 cassette. The ligation products were used to transform E. coli TG1 cells, and the transformants were selected on LB plates containing tetracycline.

\section{Measurement of Ampicillin Resistance of Cells Expressing the} $\beta$-Lactamase Fusion Proteins

The ampicillin resistance of individual E. coli TG1 cells containing various fusion plasmids was determined by growing the cells in LB to mid-log phase $\left(3 \times 10^{8}\right.$ cells $\left./ \mathrm{ml}\right)$. The cells were then washed in an equal volume of fresh medium and plated onto the same medium (solidified using $1.5 \%$ agar) containing $0,50,75$ $100,125,150,175,200$ and $225 \mu \mathrm{g} / \mathrm{ml}$ of ampicillin and $1 \mathrm{mM}$ IPTG (100-400 cells per plate). The colony numbers were determined from three independent experiments. The obtained plasmids were analyzed by DNA sequencing to identify the fusion junction sites.

\section{Measurement of the Fluorescence of the YddG-ZsGreen}

Fusions

To measure the fluorescence of the YddG-ZsGreen fusions, E. coli TG1 cells containing one of the obtained plasmids were grown in LB to mid-log phase $\left(3 \times 10^{8}\right.$ cells $\left./ \mathrm{ml}\right)$ in the presence of $1 \mathrm{~mm}$ IPTG. To measure the ZsGreen fluorescence spectrum, we used a Synergy 2 multidetection microplate reader (BioTek Instruments, Inc., USA) with a 485-nm excitation filter and a 528$\mathrm{nm}$ emission filter. The $\mathrm{OD}_{600}$ was quantified simultaneously using the same instrument. The fluorescence values were normalized to the cell density in the cultures. The fluorescence signal-to-noise ratio (arbitrary units) was calculated using the formula [(signal - noise)/noise]; where 'signal' is the fluorescence signal from the induced cells and the 'noise' corresponds to the fluorescence signal from noninduced cells. Finally, arbitrary units were indicated as a percentage, where the fluorescence of $\mathrm{Gly}_{293}$ YddG-ZsGreen was set at $100 \%$.
Preparation of Membrane Extracts

Membrane fractions were prepared according to the protocol of Rouanet and Nasser [2001]. To solubilize the obtained membrane extracts, $20 \%$ Triton X-100 was added to a final concentration of $2 \%$ and incubated at $4{ }^{\circ} \mathrm{C}$ for $1 \mathrm{~h}$. Protein concentration was determined as described by Bradford [1976].

\section{Western Blotting}

Samples were prepared by heating at $37^{\circ} \mathrm{C}$ for $5 \mathrm{~min}$ in Laemmli SDS sample buffer [Laemmli, 1970]. Proteins were separated using $0.1 \%$ SDS-12\% PAGE and were electrotransferred onto a polyvinylidene difluoride membrane using a Trans-Blot SD semidry electrophoretic transfer cell (Bio-Rad). Mouse antiE. coli $\beta$-lactamase (Abcam) and rabbit anti-RCFP sera (Clontech) were used as the primary antibodies, and goat anti-mouse and anti-rabbit alkaline phosphatase conjugates (Sigma) were used as the respective secondary antibodies. The blot was developed using 5-bromo-4-chloro-3-indolyl phosphate and nitroblue tetrazolium (Fermentas), as described by Sambrook and Russell [2001].

\section{Confocal Laser Scanning Microscopy}

To observe ZsGreen fluorescence, we diluted overnight cultures 1:50 in fresh LB media containing the appropriate antibiotic and $1 \mathrm{~mm}$ IPTG to an $\mathrm{OD}_{600}$ of 0.5 . To immobilize the bacteria, we mixed the live culture with an equal volume of $1 \%$ lowmelting-point agarose (Panreac) in phosphate-buffered saline and immediately poured the mixture onto a $0.17-\mathrm{mm}$ glass slide for jellification. The agarose was melted and cooled to $40^{\circ} \mathrm{C}$ before mixing.

The cells were imaged using an LSM510 META inverted microscope (Carl Zeiss) with a water immersion $63 \times / 1.2 \mathrm{NA}$ C-Apochromat objective. ZsGreen fluorescence was excited at $488 \mathrm{~nm}$. The emission was detected using a HFT UV/488/543/633 main beam splitter through a LP505 long-pass filter. The typical voxel size and pixel times were $0.15 \times 0.15 \times 1.0 \mu \mathrm{m}$ and $1.6 \mu \mathrm{s}$, respectively.

\section{References}

Bolivar F, Rodriguez RL, Greene PJ, Betlach MC, Heynecker HL, Boyer HW, Crosa JH, Falkow $\mathrm{S}$ : Construction and characterization of new cloning vehicles. II. A multipurpose cloning system. Gene 1977;2:95-113.

Bradford MM: A rapid and sensitive method for the quantitation of microgram quantities of protein utilizing the principle of protein-dye binding. Anal Biochem 1976;72:248-254.

Brandon LD, Goehring N, Janakimaran A, Yan AW, Wu T, Beckwith J, Goldberg MB: IcsA, a polarly localized autotransporter with an atypical signal peptide, uses the Sec apparatus for secretion, although the Sec apparatus is circumferentially distributed. Mol Microbiol 2003;50:45-60.

Broome-Smith JK, Tadayyon M, Zhang Y: Betalactamase as a probe of membrane protein assembly and protein export. Mol Microbiol 1990;4:1637-1644.

\section{Claros MG, von Heijne G: TopPred II: an im- proved software for membrane protein structure predictions. Comput Appl Biosci 1994; 10:685-686 \\ Daley DO, Rapp M, Granseth E, Melen K, Drew $\mathrm{D}$, von Heijne G: Global topology analysis of the Escherichia coli inner membrane pro- teome. Science 2005;308:1321-1323. \\ Deber CM, Wang C, Liu LP, Prior AS, Agrawal S, Muskat BL, Cuticchia AJ: TM Finder: a prediction program for transmembrane pro- tein segments using a combination of hydro- phobicity and nonpolar phase helicity scales. Protein Sci 2001;10:212-219. \\ Díaz-Mejía JJ, Babu M, Emili A: Computational and experimental approaches to chart the Escherichia coli cell-envelope-associated proteome and interactome. FEMS Microbiol Rev 2009;33:66-97.}

Doroshenko V, Airich L, Vitushkina M, Kolokolova A, Livshits V, Mashko S: YddG from Escherichia coli promotes export of aromatic amino acids. FEMS Microbiol Lett 2007;275: 312-318.

Feilmeier BJ, Iseminger G, Schroeder D, Webber H, Phillips GJ: Green fluorescent protein functions as a reporter for protein localization in Escherichia coli. J Bacteriol 2000;182: 4068-4076.

Heddle C, Mazaleyrat SL: Development of a screening platform for directed evolution using the reef coral fluorescent protein ZsGreen as a solubility reporter. Prot Eng Des Sel 2007;20:327-337.

Hirokawa T, Boon-Chieng S, Mitaku S: SOSUI: classification and secondary structure prediction system for membrane proteins. Bioinformatics 1998;14:378-379.
Airich/Tsyrenzhapova/Vorontsova/ Feofanov/Doroshenko/Mashko 
Hofmann K, Stoffel W: Tmbase - a database of membrane spanning proteins segments. Biol Chem Hoppe-Seyler 1993;347:166

Kiefer D, Kuhn A: YidC as an essential and multifunctional component in membrane protein assembly. Int Rev Cytol 2007;259:113138.

Krogh A, Larsson B, von Heijne G, Sonnhammer EL: Predicting transmembrane protein topology with a hidden Markov model: application to complete genomes. J Mol Biol 2001; 305:567-580.

Kuhn A: From the Sec complex to the membrane insertase YidC. Biol Chem 2009;390:701706.

Kyte J, Doolittle RF: A simple method for displaying the hydropathic character of a protein. J Mol Biol 1982;157:105-132.

Laemmli UK: Cleavage of structural proteins during the assembly of the head of bacteriophage T4. Nature 1970;227:680-685.

Luirink J, von Heijne G, Houben E, de Gier J-W: Biogenesis of inner membrane proteins in Escherichia coli. Annu Rev Microbiol 2005; 59:329-355.
Manoil C: Analysis of membrane protein topology using alkaline phosphatase and $\beta$-galactosidase gene fusions. Methods Cell Biol 1991;34:61-75.

Matz MV, Fradkov AF, Labas YA, Savitsky AP, Zaraisky AG, Markelov ML, Lukyanov SA Fluorescent proteins from nonbioluminescent Anthozoa species. Nat Biotechnol 1999; 17:969-973.

Rapp M, Drew D, Daley DO, Nilsson J, Carvalho T, Melén K, de Gier J-W, von Heijne G: Experimentally based topology models for $E$. coli inner membrane proteins. Protein Sci 2004;13:937-945.

Rouanet C, Nasser W: The PecM protein of the phytopathogenic bacterium Erwinia chry santhemi, membrane topology and possible involvement in the efflux of the blue pigment indigoidine. J Mol Microbiol Biotechnol 2001;3:309-318.

Sambrook J, Russell DW: Molecular Cloning: A Laboratory Manual, ed 3. Cold Spring Harbor, Cold Spring Harbor Laboratory Press, 2001.

-Santiviago CA, Fuentes JA, Bueno SM, Trombert AN, Hidalgo AA, Socias LT, Youderian P, Mora GC: The Salmonella enterica sv. Typhimurium $s m v A, y d d G$ and $o m p D$ porin genes required for the efficient efflux of methyl viologen. Mol Microbiol 2002;46: 687-698.
Sutcliffe JG: Complete nucleotide sequence of the Escherichia coli plasmid pBR322. Cold Spring Harb Symp Quant Biol 1979;43:7790.

Urbanus ML, Fröderberg L, Drew D, Bjork P, de Gier JWL, Brunner J, Oudega B, Luirink J: Targeting, insertion, and localization of Escherichia coli YidC. J Biol Chem 2002;277: 12718-12723.

Von Heijne G: The distribution of positively charged residues in bacterial inner membrane proteins correlates with the transmembrane topology. EMBO J 1986;5:30213027.

Xie K, Dalbey RE: Inserting proteins into the bacterial cytoplasmic membrane using the Sec and YidC translocases. Nat Rev Microbiol 2008; 6:234-244.

Yuan J, Zweers JC, van Dijl JM, Dalbey RE: Protein transport across and into cell membranes in bacteria and archea. Cell Mol Life Sci 2010;67:179-199. 